\title{
Breast Cancer and Pregnancy: Experience of Maternity and Neonatology Center of Tunis-Tunisia
}

Achour Radhouane ${ }^{1 *}$, Magdoud Khawla ${ }^{1}$, Hmila Tarek ${ }^{1}$, Ben Jemaa Nadia², Chanoufi Mohamed Badis ${ }^{3}$, Mahjoub Sami ${ }^{4}$, Khila Mehdi $^{3}$, Chelly Dalenda ${ }^{5}$, Malek Monia ${ }^{6}$, Rzega $\mathrm{Hedi}^{6}$ and Neji Khaled ${ }^{1}$

${ }^{1}$ Emergency Department of Maternity and Neonatology Center, Faculty of Medicine of Tunis, El-Manar University of Tunis, Tunisia ${ }^{2}$ Fetopathology Department of Maternity and Neonatology Center, Faculty of Medicine of Tunis, El-Manar University of Tunis, Tunisia ${ }^{3}$ Department "C" of Maternity and Neonatology Center, Faculty of Medicine of Tunis, El-Manar University of Tunis, Tunisia

${ }^{4}$ External consultation service of Maternity and Neonatology Center, Faculty of Medicine of Tunis, El-Manar University of Tunis, Tunisia

${ }^{5}$ Department "A" of Maternity and Neonatology Center, Faculty of Medicine of Tunis, El-Manar University of Tunis, Tunisia

${ }^{6}$ Department "B" of Maternity and Neonatology Center, Faculty of Medicine of Tunis, El-Manar University of Tunis, Tunisia

\begin{abstract}
Objectives: This document aims to identify the clinical, therapeutic, and prognostic features of this association and to provide the up-to-date management.

Methods and materials: Our study design is retrospective based on 25 cases of pregnancy-associated breast cancer, carried out at the Maternity and Neonatology Center of Tunis over a period of 10 years, between January 2001 and December 2013.

Results: The mean age of the patients was 35.84 years. Breast cancer was diagnosed during pregnancy in fourteen patients and after delivery in eleven patients. Pathohistological diagnosis was established by ultrasoundguided biopsy and surgical biopsy in fourteen and seven patients, respectively. The most common histological type was infiltrating ductal carcinoma ( $96 \%$ of cases). Pregnancy was completed in nine patients. The mean gestational age at which delivery occurred was 35.4 weeks. Twenty patients underwent surgical treatment by mastectomy and breast-conserving surgery (lumpectomy) associated with axillary lymph node dissection was performed in three cases. Chemotherapy was allowed during pregnancy, and was given to 23 patients. On the other hand, radiotherapy, antiestrogens (Tamoxifene) and targeted therapies (Herceptin) must be postponed after delivery because of their teratogenic effects. After a 5-year follow-up, two of eighteen patients died and six were lost to follow-up.

Conclusion: The poor prognosis of the pregnancy-associated breast cancer is no longer attributed to pregnancy but rather to the young age of the patients and the delay in the diagnosis of cancer. Treatment should be started promptly during pregnancy.
\end{abstract}

Keywords: Cancer; Breast; Pregnancy; Treatment; Prognosis

\section{Introduction}

Breast cancer is common, and is currently considered the first cancer in women [1]. In Tunisia, it accounts for about $30 \%$ of all female cancers [2]. Late diagnosis still exists. In 2010, about half of women with breast cancer had initial tumors larger than $35 \mathrm{~mm}$ in diameter. The term "pregnancy-associated breast cancer" is used if this cancer is diagnosed during pregnancy and up to one year after giving birth. This association was considered for a long time to be of rapid evolution and unfavorable prognosis. However, it seems that the prognosis of breast cancer is not much aggravated by pregnancy itself as by the delay in diagnosis and management. We report in this retrospective study a series of 25 patients with pregnancy-associated breast cancer (PABC) recorded over 10 years at the Tunis maternity and neonatal center (TMNC).

\section{Materials and Methods}

We present the results of a retrospective study involving 25 cases of PABC in the three obstetrics and gynecology departments A, B and C. The study period spans 10 years between 1 January 2001 and 31 December 2013.

\section{Inclusion criteria}

All breast cancers diagnosed during pregnancy or one year after childbirth.

\section{Exclusion criteria}

Breast cancers diagnosed outside the above-mentioned period. We had access to patient records with a PABC in the archives of the three hospital departments (A-B-C) of the TMNC. We attempted to identify epidemiological, clinical, para-clinical (echo-mammography, pathology), therapeutic and prognostic characteristics.

Therefore, we called the patients (and or their families in some cases).

\section{Results}

The mean age of the patients was 35.84 years (26 years- 48 years). The mean age of the menarche was 12.6 years. Two patients had a familial history of cancer; one of the patient's mother had infiltrating ductal carcinoma, while the second patient's paternal cousin and

*Corresponding author: Achour Radhouane, Emergency Department of Maternity and Neonatology Center, Faculty of Medicine of Tunis, El-Manar University of Tunis, Tunisia, Tel: 21698549398; E-mail: radhouane.a@live.com

Received May 05, 2017; Accepted May 10, 2017; Published May 15, 2017

Citation: Radhouane A, Khawla M, Tarek H, Nadia BJ, Badis CM, et al. (2017) Breast Cancer and Pregnancy: Experience of Maternity and Neonatology Center of Tunis-Tunisia. J Cancer Sci Ther 9:445-450. doi: 10.4172/1948-5956.1000457

Copyright: (ㅇ 2017 Radhouane A, et al. This is an open-access article distributed under the terms of the Creative Commons Attribution License, which permits unrestricted use, distribution, and reproduction in any medium, provided the original author and source are credited. 
maternal cousin were followed for malignant breast pathology.

The patients were classified in terms of their gravidity. Six of them were primigravidae, whereas eight patients were multiparous and eleven had 2-3 deliveries. The mean age at first birth in our population was 29.84 years ( 18 years- 43 years). Apart from the six primigravidae, all of our patients had breast-fed during their previous pregnancies. In our series, 21 patients ( $84 \%$ of the cases) consulted about breast lump noted in the breast self-examination. Two patients consulted about inflammatory breast cancer. The mean size of the palpated lump was $38 \mathrm{~mm}$ (Table 1). The diagnosis was established during pregnancy in 14 patients and postpartum in 11 patients (Table 2).

All patients underwent preoperative radiological assessment: ultrasonography (22 cases), bilateral mammography (8 cases), ultrasonography + mammography $(7$ cases) and breast MRI (1 case). We notice that between 2001 and 2006, no mammogram was carried out during pregnancy due to the possibility of the risk of fetal irradiation. After that period, eight pregnant patients were able to benefit from mammography during pregnancy by means of abdominal protection as recommended by the last articles published in the medical literature. The pathohistological diagnosis was obtained by ultrasoundguided biopsy in fourteen patients. A lumpectomy with frozen section margin evaluation was conducted in seven cases and surgical biopsy in four cases. The most common pathohistological type in our series was infiltrating ductal carcinoma ( $96 \%$ of cases). Only one patient had a carcinoma in situ of high grade comedocarcinoma type. The histoprognostic score of SCARF BLOOM-RICHARDSON was grade III in 14 patients, grade II in 8 patients, and grade I in 2 patients. The PABC metastases report included: A chest $\mathrm{x}$-ray with no suspicious anomalies: 24 cases.

-An abdomino-pelvic ultrasound was normal in 22 patients. A benign ovarian cyst was detected ( 2 cases) and a secondary multinodular liver ( 1 case). Bone scintigraphy for detecting bone metastases: 16 cases were positive. In one patient, it showed a moderate increased uptake at T12 level, and a heterogeneous increased uptake of the spine and the

\begin{tabular}{|c|c|c|}
\hline Patient & Lump Position & Lump measurement \\
\hline 1 & Right upper outer quadrant (UOQ) & $3 \mathrm{~cm}$ \\
\hline 2 & Left upper outer quadrant (UOQ) & $10 \times 9 \mathrm{~cm}$ \\
\hline 3 & Right lower outer quadrant (LOQ) & $17 \mathrm{~mm}$ \\
\hline 4 & Right upper inner quadrant (UIQ) & $2 \mathrm{~cm}$ \\
\hline 5 & Left lower outer quadrant (LOQ) & $2 \mathrm{~cm}$ \\
\hline 6 & Left upper outer quadrant (UOQ) & $4 \mathrm{~cm}$ \\
\hline 7 & Right upper outer quadrant (UOQ) & $3 \mathrm{~cm}$ \\
\hline 8 & Right upper inner quadrant (UIQ) & $5 \mathrm{~cm}$ \\
\hline 9 & Left upper outer quadrant (UOQ) & $4 \mathrm{~cm}$ \\
\hline 10 & Left upper inner quadrant (UIQ) & $3 \times 4 \mathrm{~cm}$ \\
\hline 11 & Right upper outer quadrant (UOQ) & $4 \mathrm{~cm}$ \\
\hline 12 & Right upper outer quadrant (UOQ) & $3 \mathrm{~cm}$ \\
\hline 13 & Left upper outer quadrant (UOQ) & $6 \mathrm{~cm}$ \\
\hline 14 & Right upper outer quadrant (UOQ) & $7 \mathrm{~cm}$ \\
\hline 15 & Left upper outer quadrant (UOQ) & Two lumps: $2.5 \mathrm{~cm} ; 2.2 \mathrm{~cm}$ \\
\hline 16 & Right upper inner quadrant (UIQ) & $2.5 \mathrm{~cm}$ \\
\hline 17 & Left lower inner quadrant (LIQ) & $7 \mathrm{~cm}$ \\
\hline 18 & Conjunction of left upper quadrants & $4 \mathrm{~cm}$ \\
\hline 19 & Right upper inner quadrant (UIQ) & $5 \mathrm{~cm}$ \\
\hline 20 & Left upper outer quadrant (UOQ) & $4 \mathrm{~cm}$ \\
\hline 21 & Left upper outer quadrant (UOQ) & $5 \mathrm{~cm}$ \\
\hline
\end{tabular}

Table 1: Clinical examination of patients.

\begin{tabular}{|c|c|}
\hline Patient & Time of diagnosis \\
\hline 1 & 4 months postpartum \\
\hline 2 & 33 gestational weeks +1 day \\
\hline 3 & 28 gestational weeks +2 days \\
\hline 4 & 24 gestational weeks +4 days \\
\hline 5 & 14 gestational weeks \\
\hline 6 & 29 gestational weeks \\
\hline 7 & 29 gestational weeks \\
\hline 8 & 9 months postpartum \\
\hline 9 & 5 months postpartum \\
\hline 10 & 12 gestational weeks \\
\hline 11 & Postpartum \\
\hline 12 & 34 gestational weeks \\
\hline 13 & Postpartum \\
\hline 14 & Postpartum \\
\hline 15 & 22-23 gestational weeks \\
\hline 16 & 26 gestational weeks +4 days \\
\hline 17 & Postpartum \\
\hline 18 & 23 gestational weeks \\
\hline 19 & 32 gestational weeks \\
\hline 20 & 18 gestational weeks \\
\hline 21 & 32 gestational weeks \\
\hline 22 & Postpartum \\
\hline 23 & 9 months postpartum \\
\hline 24 & 4 months postpartum \\
\hline 25 & 6 months postpartum \\
\hline
\end{tabular}

Table 2: Gestational age or the period when diagnosis has been established.

rib cage in another. The rest of the exams were within normal limits. One patient underwent a chest-abdomen-pelvis and cerebral CT which showed bilateral pleural effusion of great quantity and diffuse bone metastases.

\section{Obstetrical management}

Breast cancer was diagnosed during pregnancy in 14 patients. Pregnancy was continued in 9 cases (68\%), while five patients underwent a therapeutic termination of pregnancy (Table 3). The termination of pregnancy occurred after cervical ripening with Misoprostol (Cytotec) and then the labor was induced by oxytocin. Four of the fetuses were stillborn. For the 9 patients in whom the pregnancy was continued, the delivery route was vaginal in 5 cases and caesarean section in 4 cases. The mean gestational age at which delivery occurred was 35.4 weeks, ranging from 31 and 39 weeks.

\section{Oncologic management}

Surgical treatment: Surgical treatment was performed in 23 patients (92\%) and involved either radical surgery or Patey surgery in 20 cases and breast-conservative surgery (lumpectomy) in 3 cases. Among the twenty patients who had mastectomy, ten patients underwent Patey surgery after first chemotherapy, and ten were first operated.

\section{Medical treatment}

Chemotherapy: It was administered in 23 patients either in neoadjuvant (12 cases) or as adjuvant therapy after surgery (11 cases). The most commonly used protocol was FEC (5 FU, Epirubicin, Cyclophosphamide). Five patients received chemotherapy during pregnancy. No chemotherapy- related malformations or abnormalities were noted at the birth of newborns. For all these patients as well as those who received chemotherapy in the postpartum, breastfeeding was absolutely contraindicated. 


\begin{tabular}{|c|c|c|c|}
\hline Patients & Induction & Gestational age & Delivery route \\
\hline 2 & $\begin{array}{c}\text { Cervical Ripening } \\
\text { Prepidyl Gel }\end{array}$ & 37 Weeks & Vaginal \\
\hline 3 & $\begin{array}{c}\text { Cervical Ripening } \\
\text { Prepidyl Gel }\end{array}$ & 38 Weeks & Vaginal \\
\hline 4 & $\begin{array}{c}\text { Cervical Ripening } \\
\text { Prepidyl Gel }\end{array}$ & 37 Weeks & Vaginal \\
\hline 6 & Cesarean \\
\hline 7 & $\begin{array}{c}\text { Cesarean for iatrogenic } \\
\text { prematurity }\end{array}$ & 34 Weeks+2 Days & Cesarean \\
\hline 12 & $\begin{array}{c}\text { Cervical Ripening } \\
\text { Prepidyl Gel }\end{array}$ & 34 Weeks & Vaginal \\
\hline 16 & $\begin{array}{c}\text { Tocolysis Failure } \\
\text { Precious Pregnancy }\end{array}$ & 31 Weeks+4 days & Cesarean \\
\hline 19 & $\begin{array}{c}\text { Cervical Ripening } \\
\text { Prepidyl Gel }\end{array}$ & 35 Weeks & Vaginal \\
\hline 21 & Elective cesarean & 39 Weeks & Cesarean \\
\hline
\end{tabular}

Table 3: Gestational age when delivery occurred.

Radiotherapy: It is contraindicated during pregnancy because of the risk of fetal irradiation.

Hormone therapy and targeted therapies: In our series, and in view of the teratogenic effect reported in the literature, hormone therapy and immunotherapy were never indicated in patients with tumors expressing hormone receptors or HER 2 neu during pregnancy. On the other hand, these therapies were prescribed after childbirth.

Follow-up: After a follow-up of 5 years:

Twelve patients had no metastases during their follow-up. Two of them had a local recurrence treated first by surgery and secondly by chemotherapy. Six patients had secondary metastatic sites (hepatic in 2 cases and osseous in 4 cases), two of them died. Two patients had metastases and died a few months after delivery.

\section{Discussion}

\section{Epidemiology}

Breast cancer is the most common cancer in women. Its incidence rate in the world population varies from 15 to 20 per 100,000 women [1]. In Tunisia, breast cancer is the first cancer of women, accounting for $30 \%$ of female malignancies, with 2000 new cases annually [3]. In our country, on average, the age at which diagnosis of breast cancer is made is roughly 50 years, 10 years younger than the Western series. Besides, about $10 \%$ of patients are under the age of 35 [4,5]. Pregnancyassociated breast cancer (PABC) complicates between 1 per 10,000 and 1 per 3,000 pregnancies [6]. The estimated incidence of $\mathrm{PABC}$ is $0.2 \%$ to $3.8 \%[7,8]$. In Van Calsteren's study of 215 cases, $46 \%$ of cancers were associated with pregnancy: the first cancer associated with pregnancy [7]. In Tunisia, an ISA survey of 290 patients showed that this association affects about $20 \%$ of women of childbearing age (3). The average age of PABC is 33 years (23-47). In the European records, the average age is about 33 years (24-43 years) [8]. In our series, the average age of onset is 35.8 years ranging from 26 to 48 years.

\section{The clinical examination}

Tumor size and shape: The pregnancy-associated breast cancer $\mathrm{PABC}$ was discovered at a late stage with a pretty large size that reached $9 \mathrm{~cm} \times 7 \mathrm{~cm}$ in our series. The changes of physiological adaptations of pregnancy make breast exam difficult. For Giacalone et al. 58\% of cancers were less than $5 \mathrm{~cm}$ in size, and staged as following: T0 stage:
$0 \%$, stage $\mathrm{T} 1: 28.3 \%$, stage $\mathrm{T} 2: 43.2 \%$. Moreover, they report an increase in the size of the tumor, which is $3.5 \mathrm{~cm}$ during pregnancy and falls to 2 cm outside pregnancy [9].

Metastases to the nodes: Involvement of the axillary lymph nodes is frequent during pregnancy. It concerns $56-89 \%$ of the parturientes against $38 \%$ to $54 \%$ of the non-pregnant patients [10].

Clinical stage: Sixty-five to ninety percent of cancers in pregnant women are diagnosed at stage II and III, compared with $45 \%$ to $66 \%$ in non-pregnant controls $[11,12]$. In our series, at the initial presentation, thirteen patients had a locally advanced stage of breast cancer, ten cases at stage T3 N1 Mx, and three cases at the stage of T4D N1Mx;

\section{Diagnostic tests}

\section{Radiological examinations}

The Mammography: Mammography with lead protective shield represents a low risk for the fetus [13]. A dose of $200 \mathrm{mGy}$ to $400 \mathrm{mGy}$ is delivered during mammography. This represents an exposure of less than $50 \mathrm{mrad}(0.5 \mathrm{~Gy})$ for the embryo/fetus. The threshold level of 10 $\operatorname{rad}(100 \mathrm{mGy})$ increases the risk of fetal malformations by $1 \%$ [14]. Bilateral mammography should be routinely performed in pregnant women if a malignant lesion is suspected $[15,16]$. It may be limited to a mediolateral oblique (MLO) view of each breast in case high breast density is present. The quality of the images should be optimal, particularly in terms of contrast, in order to detect microcalcifications (16). In the series of Yang et al. [17] and Ahn [18], the sensitivity of mammography done in breasts of a pregnant was $90 \%$ and $86.7 \%$ respectively.

The breast ultrasonography +++: This is the first-line indicated imaging study for breast cancer associated with pregnancy. Its sensitivity is higher than that of mammography and reaches $100 \%$ in some series [16,19]. In the Yang series [17], 100\% of the tumors and axillary masses were identified in eighteen out of twenty patients with breast cancer during pregnancy, and for Ahn et al. the sensitivity of ultrasound reached $100 \%$ [18].

The mammary MRI: Mammary MRI uses Gadolinium as a contrast agent, which crosses the placenta. Therefore, it is responsible for malformations in animal models [20,21]. According to the recommendations of the European Society of Urogenital Radiology, MRI with the use of Gadolinium is acceptable. No complications during pregnancy having been reported [22]. During lactation, MRI is possible but breast milk should be discarded for up to 48 hours after the imaging because Gadolinium is excreted via milk [23]. Mammary MRI is only recommended in case multifocal cancer is suspected. This risk should be clearly explained to the patient as well as the risk of fetal irradiation $[22,23]$.

\section{Histological diagnosis}

The histological diagnosis can be established either by fine needle aspiration, or by surgical microbiopsy.

Fine needle aspiration: Fine-needle aspiration is not contraindicated during pregnancy even if there is a high risk of false positives and false negatives [16]. The use of this method exposes the risk of Blades due to the frequency of lobular hyperplasia with its possible aspects of nucleolus hypertrophy and lack of uniformity in size [24].

\section{Percutaneous biopsy with Tru-Cut:}

Tru-cut (core core cutting needle) biopsy: In this technique, small 
pieces of tissue samples are obtained by cutting the tissue with special biopsy needles. Tru-cut is the most commonly used percutaneous needle biopsy technique. Ultrasound-guided biopsy is the standard examination in the diagnostic evaluation of a breast lump in a pregnant or lactating woman. It can be performed under local anesthesia with a sensitivity and specificity of roughly $90 \%[23,25]$. The risks of bleeding, infection, or even fistulae in this procedure are relatively frequent. Dominici found no complications among 67 patients diagnosed with breast cancer with this interventional technique. Breastfeeding should be discontinued the days preceding the procedure to minimize glandular congestion and reduce the risk of hematoma and fistula [26]. In our series, the diagnosis of malignancy was established by ultrasound-guided biopsy in 14 patients.

\section{Histopathological features}

Histological type: The histopathological and immunohistochemical characteristics of breast cancer associated with pregnancy are identical to those of breast cancer occurring in non-pregnant young women [27].

The histologic grading: The majority of breast cancers occurring during pregnancy (40\% to $95 \%$ ) are of grade II or III with vascularlymphatic emboli [13]. In this context, Middleton [11] found $84 \%$ of PABC were low-differentiated (grade II and grade III), while it is $96 \%$ according to Ring et al. [28]. In our series, fourteen cases had III and eight cases of grade II. Only two patients had grade I breast cancer.

Node involvement: Axillary node involvement appears to be more common in PABC, with incidence ranging from $53 \%$ to $71 \%[11,12]$. In our series, 15 patients (60\% of the cases) had their axillary lymph nodes invaded.

Tumor size: Tumor size is an important prognostic factor, it is independent of all of the others. It is well known that tumor size is more important in PABC [13]. For Beadle et al. it is $3.5 \mathrm{~cm}$ on average and $2 \mathrm{~cm}$ outside pregnancy [29]. This average size is much higher in our series reaching $4.6 \mathrm{~cm}$ ranging from $2 \mathrm{~cm}$ to $9 \mathrm{~cm}$.

Hormone receptors: During pregnancy, hormone receptors are positive in less than $30 \%$ of tumors [30]. This weak receptor expression would be specific to pregnant women. In the Middleton series, $28 \%$ of tumors had positive receptors by immunohistochemistry compared to $45 \%$ in young women [11].

Overexpression of HER 2 Neu: Twenty-eight to $58 \%$ of tumors in pregnant women overexpress HER 2 Neu [30].

\section{Treatment}

\section{Locoregional treatment}

Breast surgery: Breast surgery retains all of its indications during pregnancy, regardless of gestational age, as well as in postpartum. It must be carried out in a multidisciplinary team of surgeons, obstetricians, and anesthetists to ensure fetal and maternal well-being during the perioperative period [31]. Patey's mastectomy, such as conservative surgery and axillary surgery, can be performed during pregnancy without compromising fetal development by following the same recommendations as in non-pregnant women $[12,13]$. The adjuvant chemotherapy that is allowed during pregnancy should be given during this interval (surgery-radiotherapy). Dominici operated on 67 pregnant women, 47 of them had a radical treatment and 20 received conservative treatment. The lumpectomy did not seem to increase the risk of postoperative complications [26]. Cardonick reported a study of 130 parturients, parturient treated with breast cancer without significant postoperative complications [27].

Particularities related to pregnancy: Breast surgery during pregnancy is complicated due to the hypervascularization of the gland during this period. Therefore, it imposes proper hemostasis and lymphostasis. Similarly, physiological changes limit the techniques of breast reconstructive surgery. In the case of mastectomy, breast reconstruction must always be carried out in a second stage because of the increase in contralateral breast volume during pregnancy [31].

External radiotherapy: The main effects of radiotherapy during the preimplantation period (from conception to 10 days) are represented by embryonic death and malformation risk during the embryonic phase (days 10-14 up to 8 weeks). The most described malformations are central nervous system abnormalities. Its incidence is $20 \%$ when exposure to irradiation is at $18 \mathrm{~Gy}$, and $100 \%$ when exposure is $100 \mathrm{~Gy}$. Radiation therapy also exposes children to the risk of radiation-induced malignancies in childhood [14]. In case of conservative treatment, radiotherapy will be postponed after delivery. Annane [32] reported sixteen patients undergoing breast cancer surgery during pregnancy, ten of whom received conservative treatment. No local recurrence with delayed radiotherapy after delivery was observed with a mean followup of 87 months.

\section{Systemic treatment}

Chemotherapy: When chemotherapy is administered in the first trimester, the rate of malformations is $14 \%$ to $19 \%$, and the rate dropped to $1.3 \%$ when chemotherapy is introduced in the second or third trimester [16,23]. Cardonick [27] reported a $3.8 \%$ of fetal malformations in his study, which included 130 pregnant women with breast cancer, 104 of whom received chemotherapy. That incidence rate was similar to the rate found in the general population. There are no pharmacodynamic studies available on the administration of agents, cytotoxic drugs in pregnant women. The experts' recommendations are to administer the Cytotoxic agents at the same doses as in non-pregnant women with Breast cancer $[16,27]$. The recommendation may be to use epirubicin at a dose of $100 \mathrm{mg} / \mathrm{m}^{2}$. A protocol based on Anthracylins (up to 100 $\mathrm{mg} / \mathrm{m}^{2}$ but $50 \mathrm{mg} / \mathrm{m}^{2}$ in most studies) appears to be prescribed without major materno-fetal consequences $[6,8,27]$.

Hormonotherapy: A literature review in 2004 (33) focused on pregnancies under tamoxifen and reported a fetal malformation rate of $20 \%$, such as Golden hard syndrome, genital ambiguity, craniofacial malformation, and Pierre Robin syndrome. The prescription of tamoxifene is contraindicated throughout pregnancy [8].

Targeted therapies: Expression of HER2 is important in the epithelial tissue of the fetal renal tubule. Trastuzumab crosses the placental barrier $[33,34]$. Therefore, Trastuzumab is contraindicated due to adverse effects.

\section{Therapeutic termination of pregnancy (TTP)}

Termination of pregnancy has long been considered a rational way to improve prognosis. However, several studies have recently shown that there is no evidence that TTP improves the prognosis of PABC [26]. The TTP is indicated if the continuation of the pregnancy leads to a delay in management that might be prejudicial to the maternal prognosis. We cite two situations:

1. Breast cancer discovered at a late stage in the first trimester of pregnancy which is a hindrance to using different therapeutic modalities without risk, such as radiotherapy and targeted therapies. 
2. Estimated survival is less than the duration of pregnancy

\section{The assessment of metastases}

Due to late diagnosis, the risk of metastasis is 2.5 times higher in pregnant women. The sites are mainly the liver, bones, lung, and sometimes placenta [15]. It is, therefore, recommended to carry out a thorough scan to detect the metastases as performed outside pregnancy [13]. The metastases assessment in our series consisted of a chest $\mathrm{x}$-ray ( 24 cases), abdominal ultrasound ( 22 cases) and bone scintigraphy in 16 patients.

The benefits of bone scintigraphy should be weighed against risks of this procedure. The doses delivered to the fetus are arranged in ascending order of $0.0063 \mathrm{mGy}$ at the level of the uterus, $0.0064 \mathrm{mGy}$ for a fetus of 8 weeks and $0.0026 \mathrm{mGy}$ for a fetus of 18 weeks.

Bone scintigraphy should usually be performed after childbirth. It is only justified during pregnancy if there is a strong suspicion of bone localization, which may alter the therapeutic management.

In our series, a bone scintigraphy for secondary osseous sites was performed in 16 cases, including two cases during pregnancy.

\section{Tumor markers}

The serum levels of tumor markers evaluate the aggressiveness of a tumor, its progression and/or therapeutic effectiveness. The markers used in breast cancer are CA15-3 and ancillary test of carcinoembryonic antigen (CEA). However, there is a frequent "physiological" increase in the rate of CA 15-3 at the end of pregnancy. Consequently, markers are useful in post-treatment follow-up, recurrence, or metastasis.

\section{Prognostic factors}

Maternal age: It is generally agreed that tumors occurring in young patients under the age of 35 have poor prognosis [11]. According to Gemignani and Moore [35], this poor prognosis is believed to be related to the young age of these patients rather than their status of gravidity since the pregnancy does not modify the prognosis of these tumors.

Diagnostic time: Mantovani [33] attributes the poor prognosis of this association to the delay in diagnosis of 3.4 months. Ishida et al. [10] found a delay in diagnosis of 6.2 months, with a 5- and 10-year survival rate of $65 \%$ and $55 \%$, respectively.

\section{The axillary node involvement}

Lymph node metastases are more frequent in pregnant women: $56 \%$ to $89 \%$ in pregnant women compared to $38 \%$ to $54 \%$ outside pregnancy according to the authors. Reed showed that the 5-year survival rate in pregnant women with node involvement is $50 \%$ compared with $62 \%$ in the absence of lymph node involvement. The 10-year survival rate reached $50 \%$ in $\mathrm{N}+$ women compared with $34 \%$ in $\mathrm{N}$-women [12]. Martinez-Ramos [36] has proved that the 5-year survival rate exceeds $60 \%$ when there is no metastases to lymph nodes, whereas it is $45 \%$ when the lymph nodes are invaded.

Hormone receptors: Hormone receptors constitute an important prognostic factor because of the effect of hormone receptor positivity on the response to treatment $[11,35]$. However, there is a lack of research on the impact of these receptors' negativity on prognosis.

\section{Conclusion}

The term "pregnancy-associated breast cancer" is used if this cancer is diagnosed during pregnancy and through the end of first year after giving birth. This association was considered for a long time to be of rapid evolution and unfavorable prognosis. However, it seems that the prognosis of breast cancer is not much aggravated by pregnancy itself as by the delay in diagnosis and management. Treatment should be started promptly during pregnancy.

\section{References}

1. El Saghir NS, Khalil MK, Eid T (2007) Trends in epidemiology and management of breast cancer in developing arab countries: A literature and registry analysis. Int J Surg 5: 225-233.

2. Khanfir A, Frikha M, Kallel F (2006) Breast cancer in young women in the south of Tunisia. Cancer Radiother 10: 565-571.

3. Ben Abdallah M, Zehani S, Maalej M (2009) Breast cancer in Tunisia: Epidemiologic characteristics and trends in incidence. Tunisie Medicale 87: 417-425.

4. Andersson TM, Johansson AL, Hsieh CC, Cnattingius S, Lambe M (2009) Increasing incidence of pregnancy-associated breast cancer in Sweden. Obstet Gynecol 114: 568-572.

5. Pentheroudakis G, Pavlidis N (2006) Cancer and pregnancy: Poena magna not anymore. Eur J Cancer 42: 126-140.

6. Waalen J (1991) Pregnancy poses tough questions for cancer treatment. J Nat Cancer Inst 83: 900-902.

7. Van Calsteren K, Heyns L, De Smet F (2010) Cancer during pregnancy: An analysis of 215 patients emphasizing the obstetrical and the neonatal outcomes. J Clin Oncol 28: 683-689.

8. Aebi S, Loibl S (2008) Breast cancer during pregnancy: Medical therapy and prognosis. Recent Results Cancer Res 178: 45-55.

9. Giacalone PL, Bonnier P, Laffargue F (2009) Breast cancer during pregnancy Womens Health (Lond Engl) 5: 243-249.

10. Ishida T, Yokoe T, Kasumi F, Sakamoto G, Makita M, Tomi-naga T (1992) Clinicopathologic characteristics and prognosis of breast cancer patients associated with pregnancy and lactation: Analysis of case-control study in Japan. Jpn J Cancer Res 83: 1143-1149.

11. Middleton LP, Amin M, Gwyn K, Theriault R, Sahin A (2003) Breast carcinoma in pregnant women: Assessment of clinicopathologic and immunohistochemical features. Cancer 98: 1055-1060.

12. Reed W, Hannisdal E, Skovlund E, Thoresen S, Lilleng P, et al. (2003) Pregnancy and breast cancer: A population-based study. Virchows Archiv 443: 44-50.

13. Loibl S, von Minckwitz G, Gwyn K (2006) Breast carcinoma during pregnancy International recommendations from an expert meeting. Cancer 106: 237-246.

14. Mazonakis M, Varveris $\mathrm{H}$, Damilakis $\mathrm{J}$, Theoharopoulos N, Gourtsoyiannis N (2003) Radiation dose to conceptus resulting from tagential breast irradiation. Int J Radiat Oncol Biol Phys 55: 386-391.

15. National Comprehensive Cancer Network (2008) Clinical practice guidelines in oncology: breast cancer screening and diagnosis guidelines. National Comprehensive Cancer Network, Fort Washington, PA.

16. Amant F, Deckers S, Van Calsteren K (2010) Breast cancer in pregnancy: Recommendations of an international consensus meeting. Eur J Cancer 46 3158-3168.

17. Yang WT, Dryden MJ, Gwyn K, Witman GJ, Theriault R (2006) Imaging of breast cancer diagnosed and treated with chemotherapy during pregnancy. Radiology 239: 52-60.

18. Ahn BY, Kim HH, Moon WK, Pisano ED, Kim HS, et al. (2003) Pregnancy and lactation-associated breast cancer: Mammographic and sonographic findings. J Ultrasound Med 22: 491-497.

19. Liberman L, Giess CS, Dershaw DD, Deutch BM, Petrek JA (1994) Imaging of pregnancy-associated breast cancer. Radiology 191: 245-248.

20. Aziz S, Pervez S, Khan S (2003) Case-control study of novel prognostic markers and disease outcome in pregnancy/lactation-associated breast carcinoma. Pathol Res Pract 199: 15-21. 
Citation: Radhouane A, Khawla M, Tarek H, Nadia BJ, Badis CM, et al. (2017) Breast Cancer and Pregnancy: Experience of Maternity and Neonatology Center of Tunis-Tunisia. J Cancer Sci Ther 9:445-450. doi: 10.4172/1948-5956.1000457

21. Bellin MF, Webb JA, Van Der Molen AJ (2005) Safety of MR liver specific contrast media. Eur Radiol 15: 1607-1614.

22. Webb JA, Thomsen HS, Morcos SK (2005) The use of iodinated and gadolinium contrast media during pregnancy and lactation. Eur Radiol 15: 1234-1240.

23. Vinatier E, Merlot B, Poncelet E (2009) Breast cancer during pregnancy. Eur J Obstet Gynecol Reprod Biol 147: 9-14.

24. Bottles K, Taylor RN (1985) Diagnosis of breast masses in pregnant and lactating women by aspiration cytology. Obstet Gynecol 66: 76-78.

25. Oyama T, Koibuchi Y, Core MG (2004) Needle biopsy (CNB) as a diagnostic method for breast lesions: Comparison with fineneedle aspiration cytology (FNA). Breast Cancer 11: 339-342.

26. Dominici LS, Kuerer HM, Babiera G (2010) Wound complications from surgery in pregnancy-associated breast cancer (PABC). Breast Disease 31: 1-5.

27. Cardonick E, Dougherty R, Grana G (2010) Breast cancer during pregnancy: Maternal and fetal outcomes. Cancer J 16: 76-82.

28. Ring AE, Smith IE, Ellis PA (2005) Breast cancer and pregnancy. Ann Oncol 16: $1855-1860$

29. Beadle BM, Woodward WA, Middleton LP, Tereffe W, Strom EA, et al. (2009)
The impact of pregnancy on breast cancer outcomes in women< or $1 / 435$ years. Cancer 115: 1174-1184.

30. Shousha S (2000) Breast carcinoma presenting during or shortly after pregnancy and lactation. Arch Pathol Lab Med 124: 1053-1060.

31. Ni Mhuireachtaigh R, O'Gorman DA (2006) Anesthesia in pregnant patients for non-obstetric surgery. J Clin Anesth 18: 60-66.

32. Annane K, Bellocq JP, Brettes JP, Mathelin C (2005) Infiltrative breast cancer during pregnancy and conservative surgery. Fetal Diagn Ther 20: 442-444.

33. Mantovani G, Gramignano G, Mais V (2007) Use of chemotherapy for ovarian cancer during human pregnancy: case report and literature review. Eur J Obstet Gynecol Reprod Biol 131: 238-9.

34. Press MF, Cordon-Cardo C, Slamon DJ (1990) Expression of the HER-2/neu proto-oncogene in normal human adult and fetal tissues. Oncogene 5: 953-962.

35. Gemignani ML, Petrek JA (1999) Pregnancy after breast cancer. Cancer Control 6: 272-276.

36. Martínez-Ramos D, Ferraris C (2007) Carcinoma of the breast during pregnancy. Cir Esp 82: 305-307. 